\title{
Aprendizagem na educação online: análise de conceito
}

\author{
Learning in online education: analysis of concept \\ Aprendizaje en la educación online: análisis de concepto
}

\begin{abstract}
Viviane Rolim de Holanda', Ana Karina Bezerra Pinheiro", Lorita Marlena Freitag Pagliuca"
' Universidade Federal do Ceará, Programa de Pós-Graduação em Enfermagem (Doutoranda). Fortaleza-CE, Brasil. "Universidade Federal do Ceará, Departamento de Enfermagem. Pesquisadora do Conselho Nacional de Desenvolvimento Científico e Tecnológico. Fortaleza-CE, Brasil.
\end{abstract}

Submissão: 04-07-2012 Aprovação: 28-04-2013

\section{RESUMO}

Objetivou-se clarificar o conceito aprendizagem na educação online, expresso pela literatura da área da saúde. Trata-se de um estudo de análise de conceito, baseado nas etapas do Modelo Evolucionário, destacando-se os atributos, antecedentes, consequentes e termos substitutos. No contexto do ensino online, a aprendizagem é expressa por um processo dinâmico e contínuo de construção ativa do conhecimento e aquisição de habilidades, com separação física entre alunos e professores. Entre os eventos antecedentes abordados sobressaíram: interesse e motivação para aprender; dedicação e autogerenciamento do tempo; interação e ferramentas de comunicação. Quanto aos principais consequentes evidenciados mencionam-se: autonomia do aluno; estudo independente e ativo; e construção do próprio conhecimento. Como termos substitutos predominantes sugiram aprendizagem colaborativa e autoaprendizagem. A compreensão do conceito poderá contribuir para a sua aplicação nas práticas de ensino de enfermagem em ambientes virtuais.

Descritores: Aprendizagem; Formação de Conceito; Educação a Distância; Instrução por Computador; Enfermagem.

\begin{abstract}
This work aimed at clarifying the concept learning in online education, expressed by the literature in the area of health. It is a study of analysis of concept, based on the Evolutionary Model, being highlighted attributes, background, consequents and substitute terms. Learning in the context of online education is characterized by a dynamic and continuous process of active construction of knowledge and acquisition of skills, with physical separation between students and teachers. Among the background events discussed stood out: interest and motivation in learning; dedication and time self-management; and interaction and communication tools. The main evident consequents were: student's autonomy; independent and active study; and construction of own knowledge. The prevailing substitute terms were collaborative learning and self-learning. The understanding of the concept can contribute to its implementation in nursing teaching activities in virtual environments.
\end{abstract}

Key words: Learning; Concept Formation; Distance Education; Computer-Assisted Instruction; Nursing.

\section{RESUMEN}

Este trabajo tuvo por objetivo aclarar el concepto aprendizaje en la educación online, expresado por la literatura en el área de salud. Se trata de un estudio de análisis de concepto, basado en el Modelo Evolucionario, destacándose atributos, antecedentes, consecuentes y términos substitutos. El aprendizaje, en el contexto de enseñanza online, se caracteriza por ser un proceso dinámico y continuo de construcción activa del conocimiento y adquisición de habilidades, con separación física entre alumnos y profesores. Entre los eventos antecedentes tratados se destacaron: interés y motivación en aprender; dedicación y administración del tiempo; e interacción y herramientas de comunicación. Los principales consecuentes demostrados fueron: autonomía del alumno; estudio independiente y activo; y construcción del propio conocimiento. Los términos substitutos predominantes fueron aprendizaje colaborativo y autoaprendizaje. La comprensión del concepto podrá contribuir para su aplicación en las prácticas de enseñanza de enfermería en los ambientes virtuales.

Palabras clave: Aprendizaje; Formación de Concepto; Educación a Distancia; Instrucción por Computador; Enfermería.

\section{AUTOR CORRESPONDENTE Viviane Rolim de Holanda E-mail: vivi_rolim@yahoo.com.br}




\section{INTRODUÇÃO}

Os avanços das tecnologias de informação e comunicação (TICs) e a expansão da internet romperam as barreiras geográfico-temporais de acesso à educação. Com o surgimento da web no final dos anos 1990 possibilitou-se uma nova forma de aprendizagem baseada em computador, que se difundiu impulsionada pela disponibilidade de sistemas específicos softwares para a área acadêmica - conhecidos como ambientes virtuais de aprendizagem ${ }^{(1-2)}$.

A mediação das TICs na aprendizagem tem propiciado a formação de ambientes educacionais apoiados em teorias socioconstrutivistas as quais resultaram em mudanças no processo de formação dos profissionais e, consequentemente, nas atitudes, percepções e usos dessas tecnologias nos processos de trabalho. Em acréscimo, a associação das TICs e a flexibilidade da educação a distância apontam um espaço importante para o processo de aprendizagem ${ }^{(1)}$.

As Diretrizes Curriculares Nacionais dos cursos de graduação da área da saúde recomendam a prática de estudo independente, com vistas a uma progressiva autonomia intelectual a fim de preparar o graduando para enfrentar os desafios das transformações da sociedade e do mercado de trabalho ${ }^{(3)}$. Nesse sentido, acredita-se que as TICs geram estímulos e desafios para a prática de ensino e aprendizagem.

De acordo com o Decreto 5.622/2005 que regulamenta a Lei de Diretrizes e Bases da educação nacional (LDB), a educação a distância é uma forma de ensino que possibilita a aprendizagem com a mediação de recursos didáticos sistematicamente organizados, apresentados em diferentes suportes de informação, utilizados isoladamente ou combinados, e veiculados pelos diversos meios de comunicação(4).

Contudo, a educação a distância não mais se caracteriza pela distância, porquanto a virtualidade permite encontros cada vez mais efetivos que favorecem o processo ensino/ aprendizagem. É, pois, oportuno adotar o termo educação online para o processo de ensino mediado pelas TICs em ambientes digitais de aprendizagem.

As tecnologias de informação propiciam um alto poder de interação entre os participantes rompendo com a ideia de espaço e tempo. Desta forma, nos ambientes online de aprendizagem o que era distante pode se tornar perto. A dimensão do tempo e do espaço são instituídas consoantes as necessidades, os interesses e a vontade dos aprendizes, ampliando as possibilidades da educação ${ }^{(5)}$.

Desse modo, há interesse em entender o conceito de aprendizagem no cenário da educação online que facilite compreender os fatores associados à apropriação e uso dos conhecimentos e habilidades desenvolvidos nesses ambientes, bem como os elementos que promovem e limitam o uso dessas tecnologias na educação. Neste contexto, o esclarecimento do significado, do uso e da aplicação de um conceito é um passo importante no processo de desenvolvimento de uma disciplina. Com um conceito definido pode-se caracterizar fenômenos de interesse e avaliar suas forças e seus limites além de mantê-lo útil e aplicável a um determinado contexto.

Em face do exposto, ressalta-se a relevância de analisar o conceito aprendizagem mediada na educação online, enfatizando os elementos contextuais presentes em suas definições e aplicações na enfermagem, proporcionando ampla compreensão desse conceito. Dessa forma, pode-se contribuir para sua correta utilização.

O estudo justifica-se pela necessidade de maior compreensão conceitual diante das possíveis aplicações no ensino de enfermagem, na tentativa de responder às necessidades emergentes de formação seja na graduação, na pós-graduação ou educação permanente. Logo, objetivou-se clarificar o conceito aprendizagem na educação online, expresso pela literatura da área de saúde.

\section{MÉTODO}

Trata-se de um estudo de análise de conceito baseado nas etapas do Modelo Evolucionário, no qual o destaque é a investigação indutiva e descritiva, com ênfase à natureza do conceito. Compreende o conceito como dinâmico e influenciado pelo contexto, representando uma ideia abstrata expressa pelo grupo de atributos que o constitui, no intuito de clarificá-lo e reduzir problemas conceituais existentes ${ }^{(6)}$.

As etapas desse modelo são inter-relacionadas e incluem: identificar o conceito de interesse e expressões associadas; identificar e selecionar campo apropriado para coleta de dados; realizar a coleta de dados; analisar os dados distinguindo as características do conceito, seus antecedentes, consequentes e termos substitutos; identificar caso modelo do conceito; identificar hipóteses e implicações para outros estudos ${ }^{(6)}$.

O conceito de interesse selecionado foi aprendizagem no contexto da educação online, considerando sua relevância para a prática de ensino de enfermagem. Em seguida, procedeu-se à busca na Biblioteca Virtual de Saúde (BVS), Base de Dados em Enfermagem (BDENF) e na PubMed (National Library of Medicine and National Institutes of Health), no mês de outubro de 2011, seguindo-se como descritores: aprendizagem (Learning/ Aprendizaje) e educação a distância (Education, Distance/ Educación a Distancia); aprendizagem e instrução por computador (Computer-Assisted Instruction/ Instrucción por Computador).

Os critérios de inclusão adotados foram: ser artigo completo e estar disponível eletronicamente, estar publicado nos idiomas português, inglês ou espanhol no período de 2001 a 2011, ter relação com o conceito em foco. Excluíram-se artigos repetidos, estudos que não tinham relação com o conceito em análise ou que não possuíam elementos suficientes para a análise do conceito.

Procedeu-se à leitura do título e do resumo para verificar se atendiam aos critérios de inclusão estabelecidos. Em seguida, fez-se a leitura crítica dos artigos selecionados, buscando a identificação dos elementos constituintes do conceito: atributos, antecedentes e consequentes.

A análise dos atributos essenciais que expressam a natureza do conceito foi guiada pelas questões: Como o autor define o conceito? Quais as características/atributos apontados por ele? Que ideias o autor discute sobre o conceito aprendizagem?

Para a identificação dos eventos antecedentes, ou seja, 
situações que precedem o conceito de interesse usou-se a seguinte pergunta norteadora: Que eventos contribuem para a iminência do conceito aprendizagem nos ambientes virtuais? E por fim, para evidenciar os eventos consequentes resultantes da aprendizagem, utilizou-se a questão: $\mathrm{O}$ que aconteceu como resultado da aprendizagem?

Após leitura exaustiva do material, os dados foram revisados e organizados de maneira indutiva e temática. A apresentação dos resultados está organizada em quadro e tabela com frequência absoluta. Para esclarecer e revelar o estado atual do conhecimento relacionado ao conceito em interesse, desenvolveu-se a análise dos dados.

\section{RESULTADOS}

Quadro 1 - Síntese da análise do conceito aprendizagem no contexto da educação online, segundo Método Evolucionário, 2012.

Análise do conceito aprendizagem no contexto do ensino online

Termos substitutos

Aprendizagem colaborativa

Autoaprendizagem

Aprendizagem a distância

Aprendizagem online

Aprendizagem em rede

\section{Atributos essenciais}

Construção ativa do conhecimento

Processo dinâmico e contínuo

Aquisição de habilidades motoras, cognitivas e intelectuais

Experiência assíncrona

Mudança de comportamento

Desenvolvimento de sentido crítico (gerar reflexões sobre o assunto)

Desenvolvimento de significados e conhecimento compartilhado

Estímulo às capacidades investigativas do aluno

Ressignificação de saberes

Capacidade de aprender a aprender

\section{Antecedentes}

Interação e estímulo

Interesse e motivação para aprender

Dedicação, disciplina e gerenciamento de tempo (autogestão)

Ferramentas de comunicação (assíncronas e síncronas)

Materiais didáticos organizados e informações adequadas (método ativo)

Mudança no papel do professor e do aluno

Colaboração (trabalho colaborativo)

Mediação

Ambiente de afetividade e dialógico

\section{Consequentes}

Estudo mais independente e ativo

Protagonismo e autonomia do aluno

Construção do próprio conhecimento

Atualização e produção de conhecimentos

Aprendizagem significativa e contextualizada

Liberdade para construir seu próprio percurso e ritmo de estudo

Formação de sujeitos críticos

Desenvolvimento de habilidades

Conhecimento cooperativo
Inicialmente encontrou-se 153 artigos, mas com a aplicação dos critérios de inclusão foram eliminados 121. Desse modo, a amostra do estudo compôs-se de 32 artigos. Desses, a maioria datava do período de 2007 a 2011. Quanto ao idioma, analisaram-se 23 artigos em português, cinco em inglês e quatro em espanhol.

O quadro 1 apresenta a síntese da análise do conceito, constam os atributos essenciais e os termos substitutos que estão frequentemente associados ao conceito aprendizagem, nos mais diversos contextos da educação online. E apontam-se os eventos antecedentes prevalentes para expressar a ocorrência do conceito em análise e os eventos consequentes que ocorrem como resultado do uso do conceito.

Em seguida, apresenta-se o caso modelo com a presença dos atributos críticos para o conceito aprendizagem em ambiente de ensino online.

\section{CASO MODELO}

Graduando em enfermagem matricula-se no ambiente virtual da universidade para curso online de uma determinada disciplina. No decorrer das aulas, passa a construir ativamente o seu conhecimento utilizando-se das ferramentas assíncronas, da interação e colaboração entre professor e outros alunos do curso. O estudante é incentivado a organizar melhor o seu tempo e priorizar os conteúdos a serem estudados. Ao final do semestre, o aluno adquire novas habilidades e refere que se sentiu motivado para o aprender.

\section{DISCUSSÃO}

Após leitura crítica do material analisado, percebeu-se que aprendizagem é um conceito presente em diversas disciplinas acadêmicas da área da saúde com semelhanças nas definições assumidas no uso do conceito na perspectiva do ensino online.

É importante inferir que os termos substitutos são meios alternativos de expressar o mesmo conceito, diferentemente da palavra ou expressão selecionada pelo pesquisador para focar o estudo ${ }^{(6)}$. Eles devem ser identificados, em alguma dimensão, durante a coleta de dados mediante permuta da terminologia, e compartilham dos mesmos atributos do conceito em análise.

Os atributos essenciais expressam a natureza do conceito e permitiram perceber como os autores definem o conceito, as características a ele atribuídas e as ideias que discutiam sobre a aprendizagem e a aplicação e uso no contexto da educação online.

A análise dos artigos resultou na definição do conceito de aprendizagem como processo dinâmico e contínuo de construção ativa do conhecimento e aquisição de habilidades. Evidencia-se que os atributos - processo dinâmico e construção ativa do conhecimento - foram consistentes no ambiente de ensino online.

Também, como se destaca, a aprendizagem nesse ambiente é uma experiência assíncrona que promove mudança de comportamento, reflexões críticas sobre o assunto e estimula a capacidade investigativa do aluno. 
Para fornecer uma demonstração prática do conceito e delinear as suas características essenciais buscou-se construir um caso modelo. O caso ideal deve ser universal para ilustrar claramente o conceito em interesse e possibilitar a sua aplicação efetiva(6).

No caso modelo apresentado verificam-se os atributos essenciais. Por ser um fenômeno individual que promove mudança de comportamento e desenvolvimento de significados, tal percepção é identificada no aluno ao ter maior autonomia na construção do conhecimento mediante um trabalho cooperativo, organização do seu próprio ritmo de estudo e referir motivação no processo de aprendizagem.

Como observado, os avanços nas tecnologias de informação e comunicação e a expansão da internet romperam as barreiras geográfico-temporais de acesso à educação. Os autores discutiram a aprendizagem como um processo contínuo uma vez que o conhecimento não pode ser transmitido como algo acabado, por ser fruto de uma relação/interação do sujeito com o meio. Como processo dinâmico, a aprendizagem deixa de ter caráter linear e passa a ser percebida dentro de um processo de simultaneidade expandido pelas práticas de cooperação entre os sujeitos, construídas continuamente por meio da motivação e interação ${ }^{(7)}$.

No contexto da educação online, a aprendizagem é realizada por meio da separação física entre alunos e professores. $\mathrm{O}$ aprendizado e a comunicação acontecem via recursos tecnológicos que ultrapassam a exposição oral e permitem ao aluno navegar de forma não-linear, ou seja, de acordo com sua necessidade de estudo ${ }^{(8)}$. Isto porque as ferramentas assíncronas não exigem a presença simultânea dos participantes e os acessos podem ser feitos nos horários disponíveis de cada um ${ }^{(9)}$.

Nesta concepção, os níveis de participação e interação humana são elementos críticos para o sucesso das experiências de aprendizagem, pois contribuem significativamente para a efetividade das ações educacionais apoiados em modelos mentais reflexíveis da realidade, capazes de evoluir em sucessivas e crescentes formalizações ${ }^{(1,10)}$.

Como experiência assíncrona, a aprendizagem permite o acesso independente de tempo ou lugar, atendendo às necessidades individuais dos estudantes e possibilitando ao aprendiz a decisão sobre os assuntos a serem explorados, escolha de métodos e estratégias. Desse modo, resulta em uma experiência genuína, com integração de novos conhecimentos e desenvolvimento de habilidades mediante conexão de alunos com professores e outros participantes ${ }^{(11,2)}$.

Aprender, neste sentido, é estimular as capacidades investigadoras dos alunos, ajudando-os a desenvolver competências e habilidades mentais, internalizar conceitos para lidar com a realidade, resolver problemas, tomar decisões e formular estratégias de ação ${ }^{(12-13)}$.

Segundo se observa, antecedentes são aqueles eventos ou incidentes que devem ocorrer previamente à ocorrência do conceito e que favorecem o entendimento do contexto no qual ele está sendo usado.

Consoante os artigos analisados revelaram a aprendizagem na educação online baseia-se no desenvolvimento de significados compartilhados entre os participantes, tendo a interação, a motivação e a autogestão do tempo como suportes fundamentais para o alcance de seus propósitos ${ }^{(1)}$.

No processo de aprendizagem deve-se considerar a motivação para aprender; o aluno precisa ter interesse no que está fazendo; é fundamental ter significado pessoal. Vários autores apontaram a motivação de aprender algo e a autogestão do tempo como condições propícias ao desenvolvimento da aprendizagem $^{(14)}$. Determinado estudo revelou que a aprendizagem depende mais do esforço do próprio indivíduo (autogerenciamento da aprendizagem) do que dos recursos instrucionais ${ }^{(15)}$.

Entretanto, como se ressalta, na educação online é mister potencializar a vontade dos aprendizes. $\mathrm{O}$ docente deve cativar para construir um ambiente de afetividade e estimular o diálogo por meio de ferramentas que facilitarão o aprendizado ${ }^{(5)}$. Neste contexto, a aprendizagem requer atividades didáticas enriquecedoras que incluam informação e comunicação por meio de ferramentas e recursos que auxiliem no trabalho colaborativo(16).

A utilização de ferramentas assíncronas aumenta o nível de motivação dos participantes e a realização das atividades propostas. Assim, as informações publicadas ficam disponíveis para o acesso do grupo, socializando as discussões e gerando reflexões sobre o assunto nas comunidades virtuais. Estas são concebidas com o propósito de favorecer a interação dialógica entre seus participantes no ciberespaço onde todos devem se expressar ${ }^{(9,17)}$.

Além disso, essas ferramentas de comunicação promovem a interação e a colaboração porquanto facilitam a interconexão de uma série de pessoas com a finalidade de propiciar o fluxo de informação entre elas, e também a realização de trabalhos conjuntos. Estas ferramentas de comunidade virtual em rede incluem: correio eletrônico, chat, mensagens instantâneas, blogs, wikis e fóruns ${ }^{(13)}$.

Percebe-se que a interação social propiciada pelas ferramentas síncronas e assíncronas modela o tipo de relações surgidas nesses contextos de ensino. Os níveis de participação e interação humana são elementos críticos no sucesso das experiências de aprendizagem, pois possibilitam a presença social que contribui significativamente para a efetividade das ações educacionais ${ }^{(1)}$.

Outro evento percebido foi a mudança nos papéis desempenhados pelos professores e pelos alunos, além de modificações na elaboração dos materiais didáticos direcionados aos ambientes digitais de ensino. Por não haver contato direto entre aluno e professor, se requer os conteúdos tratados de modo especial, ou seja, terem a estrutura ou organização que os torne passíveis de aprendizado a distância ${ }^{(16,18)}$.

Como consta em estudos, os materiais de ensino devem conter atividades sistematizadas que desafiem os alunos, favoreçam o diálogo e promovam a busca intuitiva. As ações do professor/tutor devem motivar o aluno a fortalecer sua autoaprendizagem e a colaborar com os outros alunos, de forma a ampliar o compromisso pedagógico ${ }^{(8,19)}$.

Nesta óptica, o professor passa a ser agente e mediador fundamental da construção do estudante, facilitador dos novos métodos de aprendizagem, encorajando os aprendizes a fazerem escolhas sensatas e promover mudança de comportamentos ${ }^{(20)}$.

Em relação aos consequentes, a sua identificação é útil 
por gerar novas propostas de pesquisa e possibilitar o entendimento ampliado do conceito de interesse. Com base nos artigos analisados, os consequentes apontam que a aprendizagem no contexto da educação online é construída buscando a informação de forma ativa, no ritmo próprio do aluno, despertando maior motivação ao estudante.

A partir dessa análise, segundo evidenciado, essas experiências estimulam a autonomia do aluno uma vez que a separação física induz os alunos a desenvolverem comportamento de gerenciamento do seu aprendizado, portanto precisam planejar os períodos e o tempo de estudo e estabelecer a prioridade dos conteúdos a serem aprofundados e revisados por eles próprios ${ }^{(21)}$.

O aluno é considerado como centro do processo de aprendizagem e tem o poder de tomada de decisões, gerenciamento de sua própria aprendizagem. Para tal, precisa interagir com outros alunos e com o professor ${ }^{(22)}$.

Conforme alguns estudos evidenciam os estudantes assumem maior responsabilidade por sua aprendizagem nos cursos via internet, pensam criticamente e participam bem mais que na modalidade presencial, e são constantemente incentivados a aprender a aprender. A ambiência dos encontros de ensino em rede favorece o acesso aos objetos de informações de maneira personalizada propiciando aos estudantes definirem a dinâmica de estudo, a aquisição de habilidades e a atualização do conhecimento ${ }^{(13,16)}$.

Dessa forma, a autonomia concedida ao aluno Ihe atribui um papel mais ativo na construção de um conhecimento compartilhado, significativo e contextualizado. A participação em espaços virtuais coletivos significa assumir a responsabilidade na construção do próprio conhecimento, permitindo a liberdade para organizar seus estudos e mobilizando com isso a vontade de aprender ${ }^{(1,21)}$. De tal modo, as tecnologias de informação e comunicação auxiliam como ferramentas de ensino de maneira atraente e a aquisição do conhecimento dependerá menos do professor.

No entanto, o ensino interativo requer do professor sensibilidade para promover modificações no pensamento do aluno à medida que os expõem a novas ideias, valorizando suas experiências e fortalecendo-os para se tornarem aprendizes críticos para aquisição de conhecimentos novos e relevantes ${ }^{(2)}$.

Pode-se afirmar, portanto, que as propostas para práticas de ensino em ambientes online devem observar os atributos essenciais e os antecedentes aqui identificados com vistas a promover uma aprendizagem significativa e mais independente, e a autonomia do aluno, como sujeito crítico, para a construção e atualização do seu próprio conhecimento.

\section{CONSIDERAÇÕES FINAIS E IMPLICAÇÕES PARA A ENFERMAGEM}

A análise do conceito pelo Método Evolucionário clarificou a compreensão do fenômeno, possibilitando o uso adequado da terminologia aprendizagem no contexto online, expressa pela literatura da área de saúde.

Foram associados, como atributos essenciais ao conceito aprendizagem na educação online, o processo dinâmico e a construção ativa do conhecimento. Os eventos antecedentes identificados foram: interação, estímulo, motivação, dedicação e autogestão do tempo. Como consequentes destacaram-se: estudo independente, autonomia do aluno e construção própria do conhecimento. Prevaleceram como termos substitutos a aprendizagem colaborativa e a autoaprendizagem.

Como apontam os artigos analisados, em um mundo conectado em redes a educação online está se expandindo, e, desse modo, afeta o processo de ensino/aprendizagem, principalmente no ensino superior. $\mathrm{O}$ ensino de enfermagem acompanha essa tendência e possibilita ao aluno buscar conhecimentos e habilidades por meios mediados e na troca com o outro em ambientes digitais de aprendizagem.

Dessa forma, levante-se a hipótese que a utilização de ambientes online pode responder as necessidades de ensino garantindo uma aprendizagem eficaz para os alunos. É possível a educação tradicional combinar a experiência desses ambientes focando a participação do aluno e construindo um modelo de aprendizagem colaborativo, ativo e transformador.

Portanto, os resultados desse estudo fornecem implicações para a educação em enfermagem, a fim de fortalecer seu próprio saber por meio da compreensão do desenvolvimento de conceitos e sua correta utilização. Contudo, recomendam-se o delineamento de outras pesquisas para aprofundar a aplicação efetiva do conceito aprendizagem relacionada aos ambientes virtuais que empregam as tecnologias da informação e da comunicação com vistas a refinar elaborações teóricas para seu uso e aplicação na enfermagem.

Por fim, destaca-se como limite do estudo o fato de terem sidos avaliados artigos publicados eletronicamente, o que pode refletir em uma revisão incompleta do conceito em análise. Considera-se, então, importante suscitar indagações sobre o conceito de interesse para responder às necessidades emergentes das práticas de ensino da enfermagem no cenário das novas mídias e tecnologias na educação e os desdobramentos que essa experiência pode possibilitar aos alunos e aos professores.

\section{REFERÊNCIAS}

1. Laguardia J, Casanova A, Machado R. A experiência de aprendizagem on-line em um curso de qualificação profissional em saúde. Trab Educ Saúde 2010;8(1):97-122.

2. Aguiar RV, Cassiani SHB. Desenvolvimento e avaliação de ambiente virtual de aprendizagem em curso profissionalizante de enfermagem. Rev Latino-Am Enferm
2007;15(6):1086-1091.

3. Brasil. Conselho Nacional de Educação. Câmara de Educação Superior. Parecer no 1113, de 7 de agosto de 2001. Diretrizes Curriculares nacionais dos cursos de graduação em enfermagem, medicina e nutrição [parecer na internet]. Diário Oficial da União 03 out 2001 [acesso em 
04 maio 2012 ]. Disponível em: http://portal.mec.gov.br/ sesu/arquivos/pdf/113301EnfMedNutr.pdf

4. Brasil. Presidência da República. Casa civil. Subchefia para assuntos jurídicos. Decreto no. 5.622, de 19 de dez de 2005. Regulamenta o art. 80 da Lei $n^{\circ}$ 9.394, de 20 de dezembro de 1996, que estabelece as diretrizes e bases da educação nacional [decreto na internet]. Diário Oficial da União 20 dez 2005 [acesso em 02 nov 2011]. Disponível em: http://www.planalto.gov.br/ccivil_03/_Ato20042006/2005/decreto/D5622.htm

5. Dias DC, Cassiani SHB. Educação de enfermagem sem distâncias - uma ruptura espaço/temporal. Rev Esc Enferm USP 2004;38(4):467-74.

6. Rodgers BL. Concept analysis: an evolutionary view. In: Rogers BL, Knafl KA. Concept development in nursing: foundations, techniques, and applications. $2^{\text {th }}$ ed. Philadelphia: Saunders; 2000. p.77-102.

7. Nunes TWN, Franco SRK, Silva VD. Como a educação a distância pode contribuir para uma prática integral em saúde? Rev Bras Educ Méd 2010;34(4):554-64.

8. Telles Filho PCP, Cassiani SHB. Creation and evaluation cycle of a distance module for nursing undergraduates, named "Medication administration". Rev Latino-Am Enferm 2008;16(1):78-85.

9. Kelmer S, Coelho-Oliveira A, Fonseca LMB. Educação a distância mediada pela internet: linfonodo sentinela, prevenção, diagnóstico precoce e biópsia - nova técnica de abordagem do câncer de mama. Radiol Bras 2007;40(4):251-54.

10. Vicente R, Victoria MS, Soares AB. Análise qualitativa da concepção de professores sobre informática na educação. Psicol Argum 2011;29(65):155-66.

11. Alvarez AG, Dal Sasso GTM. Virtual learning object for the simulated evaluation of acute pain in nursing students. Rev Latino-Am Enfermagem 2011;19(2):229-37.

12. Peixoto MAP, Brandão MAG, Santos G. Metacognition and symbolic educational technology. Rev Bras Educ Méd 2007;31(1):67-80.
13. Ramos Pérez L, Domínguez Lovaina J, Gavilondo Mariño X, Fresno Chávez C. ¿Software educativo, hipermedia o entorno educativo? ACIMED [periódico na Internet]. 2008 [acesso em 07 out 2011];18(4). Disponível em: http://scielo. sld.cu/scielo.php?script =sci_arttext\&pid =S1024-94352008001000006\&lng $=$ es\&nrm $=$ iso\&tlng $=e s$

14. Zerbini T, Abbad G. Estratégias de aprendizagem em curso a distância: validação de uma escala. Psico USF 2008;13(2):177-187.

15. Zerbini T, Abbad G. Qualificação profissional à distância: avaliação da transferência de treinamento. Paidéia 2010; 20(47):313-323.

16. Jardines Méndez JB. Educación en red: mucho más que educación a distancia.Experiencia de las universidades médicas cubanas. Educ Med Super [periódico na internet]. 2006 [acesso em 01 out 2011];20(2). Disponível em: http://bvs.sld.cu/revistas/ems/vol20_2_06/ems07206.htm

17. Mutti RMV, Axt M. Towards a stated position in pedagogical discourse mediated by virtual learning environments. Interface Comun Saúde Educ 2008;12(25):347-61.

18. Schwartzman UP, Batista KT, Alves ED. Os saberes (des) complicados para educação à distância em saúde. Comun Ciênc Saúde 2009;20(3):265-70.

19. Barilli ECVC, Ebecken NFF, Cunha GG. A tecnologia de realidade virtual como recurso para formação em saúde pública à distância: uma aplicação para a aprendizagem dos procedimentos antropométricos. Ciênc Saúde Coletiva 2011;16(Suppl.1):1247-56.

20. Perfeito JAJ, Forte V, Giudici R, Succi JE, Lee JM, Sigulem D. Desenvolvimento e avaliação de um programa multimídia de computador para ensino de drenagem pleural. J Bras Pneumol 2008;34(7):437-44.

21. Silva APSS, Pedro ENR. Autonomy in nursing students process of knowledge construction: the educational chat as a teaching tool. Rev Latino-Am Enferm 2010;18(2):210-6.

22. Quelhas MCF, Lopes MHBM, Ropoli EA. Distance learning on surgical material sterilization processes. Rev Esc Enferm USP 2008;42(4):697-705. 